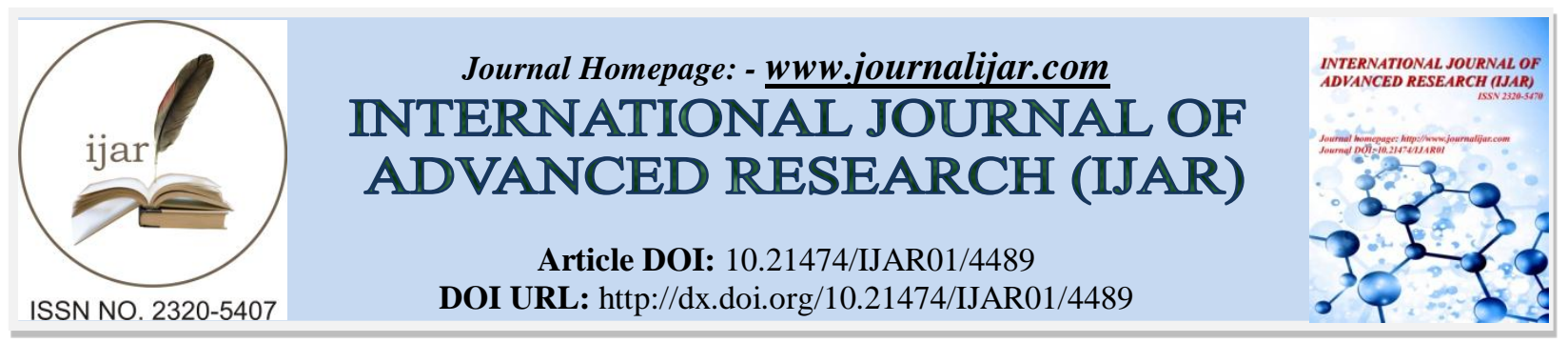

RESEARCH ARTICLE

\title{
UNIT ROOT TESTS FOR HEAVY TAILED OBSERVATIONS WITH A CHANGE POINT IN SCALE VOLATILITY
}

"BAI Zhilin and JIN Hao.

College of Science, Xi'an University of Science and Technology, Xi'an, China.

\section{Manuscript Info}

\section{Manuscript History}

Received: 15 April 2017

Final Accepted: 17 May 2017

Published: June 2017

Key words:-

Unit root test; Scale volatility; Change

points; Heavy-tailed

\section{Abstract}

This paper explores the effects of conventional unit root tests to heavy tailed errors with non-constancy scale volatility. The limiting distribution of the test statistic is derived, depending on the break in scale volatility. The results from simulations indicate that the unit root tests are potentially unreliable in the presence of such behavior and suffer some size distortions, with early negative change points in the scale.

Copy Right, IJAR, 2017,. All rights reserved.

\section{Introduction:-}

Testing for the presence of a unit root has become a problem of great concern to economists. The unit root test was originated by Dickey, Fuller (1979), who came up with the ADF test which the data generation process was autoregressive process of order $p$. Subsequently, Fuller et al. (1981), Said and Dickey (1984) and Phillips (1987) have permitted the development and applications of formal tests of unit root. In the recent literature on integrated processes increasing attention has been paid to the impact of structural changes on both size and power of unit root tests. Perron (1989) showed that the presence of a shift in the deterministic trend can reduce the power of unit root tests dramatically. Hamori and Tokihisa (1997) examined the effects of shifts in variance on the unit root tests and showed that the limiting distribution of the standard unit root test is not invariant to changes in variances. Giuseppe Cavaliere (2004) provided a general framework for investigating the effects of permanent changes in the variance of the errors of an autoregressive process on unit root tests. Furthermore, we should nevertheless bear in mind that some variables do not exist just one change. For example, Lumsdaine and Papell (1997) have considered the presence of two breaks in trend variables; Clemente et al. (1998) extended the Perron and Vogelsang (1992) statistics to the case of two changes in the mean. Leybourne and Newbold (2000a, b) proposed that unit root tests are seriously affected by the presence of relatively early breaks in the slope of the deterministic trend. For more details about this issue, we refer the reader to Joakim Westerlund(2014), Larsson and Lyhagen (2015) and Ryota Yabe (2017) among others.

All these above studies concentrated on the case where the variances of time series are finite. Many types of data from economics and finance have the same character: a heavier tail than the normal variants and it is more precise to model these heavy-tailed data with some $\kappa$-stable processes, where the index $\kappa$ can reflect the heaviness of the data. Chan and Tran (1989) proposed for a first order autoregressive process where the innovations are i.i.d which belongs to the domain of attraction of a stable law and the limiting distribution was established as a functional of a Levy process. Agnieszka Jach (2003) reported that the subsampling methodology can be used to develop unit root tests when the noise sequence is heavy-tailed with infinite variance. Horvath and Kokoszka (2003) analogously developed a residual bootstrap approximation to the distribution of a least-squares estimator of the autoregressive 
parameter when this parameter is equal to unity. Guodong Li et al. (2014) proposed a hybrid bootstrap method that approximates the enhanced Dickey-Fuller test by disturbing the residual sequence and the minimum value of the objective function and this method is efficient in handling heavy tailed sequence. When the innovations have heavy tail and dependent on the unit root test, Stelios Arvanitis (2017) proved a function limit theorem which has complex rates and limits that depend on levy process. However, the literatures suppose that the scale volatility of heavy tailed errors is constant. Therefore, the purpose of this paper is to examine the effects of a break in scale volatility on conventional unit root under heavy tailed sequences.

The paper is organized as follows. In section 2 introduces data generating process (DGP) and some crucial assumptions. The limit distribution of statistic is also derived. The finite size and power properties of the unit root test are investigated by Monte Carlo simulations in Section 3. Section 4 provided the concluding remarks.

\section{Main Result:-}

Suppose the observations $y_{t}$ follow the model with sample size $T$

where

$$
y_{t}=\rho y_{t-1}+u_{t}, u_{t}=\delta_{t} \varepsilon_{t} t=1,2, \cdots, T
$$

$$
\delta_{t}=\left\{\begin{array}{l}
\alpha, t \leq[v T] \\
\beta, t>[v T]
\end{array}\right.
$$

and $v \in[0,1] . \alpha, \beta$ are unknown values and $\left\{\varepsilon_{t}\right\}$ are I.I.D sequence satisfying the following assumption.

Assumption2.1. The strictly stationary symmetrical innovations $\varepsilon_{t}$ are in the domain of attraction of a stable law with index $1<\kappa<2$, and $E \varepsilon_{t}=0$

Our approach relies on the following results derived in Theorem 3 of Kokoszka and Wolf (2004) and the Sec. 4.4 of Resnick (1989).

Lemma2.1. If Assumption 2.1 holds, then

where

$$
\left(a_{T}^{-1} \sum_{t=1}^{[T r]} \varepsilon_{t}, a_{T}^{-2} \sum_{t=1}^{[T r]} \varepsilon_{t}^{2}\right) \stackrel{d}{\rightarrow}(U(r), V(r)) \text { in } D\left([0,1], R^{2}\right)
$$

$$
a_{T}=\inf \left\{x: P\left(\left|\varepsilon_{t}\right|>x\right) \leq T^{-1}\right\}
$$

and the random variable $U(\cdot)$ is $\kappa$-stable and $V(\cdot)$ is $\kappa / 2$-stable Levy process in [0,1]. The symbol $\stackrel{d}{\rightarrow}$ represents for convergence in distribution.

The exact definition of the Levy process $(U(r), V(r))$ appearing in the lemma1 is not required in the following, but we can recall that the $a_{T}$ can be expressed as

for some slowly varying function $L$.

$$
a_{T}=T^{1 / \kappa} L(T)
$$

Therefore, we can rewrite

$$
\delta_{t}=\alpha I_{[t \leq[T \tau]]}+\beta I_{[t>[T \tau]]} .
$$

together with Assumption 2.1 and (3), it ensures that

$$
\begin{gathered}
a_{T}^{-1} \sum_{t=1}^{[T r]} u_{t}=a_{T}^{-1} \sum_{t=1}^{[T r]}\left[\alpha I_{[t \leq[T \tau]]}+\beta I_{[t>[T \tau]]}\right] \varepsilon_{t} \\
\stackrel{L}{\rightarrow} \alpha U(r) I_{[r \leq \tau]}+\{\alpha U(\tau)+\beta[U(r)-U(\tau)]\} I_{[r>\tau]} \equiv U(\tau, r) \\
a_{T}^{-2} \sum_{t=1}^{[T r]} u_{t}^{2}=a_{T}^{-2} \sum_{t=1}^{[T r]}\left[\begin{array}{l}
\left.\alpha I_{[t \leq[T \tau]]}+\beta I_{[t>[T \tau]]}\right]^{2} \varepsilon_{t}^{2} \\
\stackrel{L}{\rightarrow} \alpha^{2} V(r) I_{[r \leq \tau]}+\left\{\alpha^{2} V(\tau)+\beta^{2}[V(r)-V(\tau)]\right\} I_{[r>\tau]} \equiv V(\tau, r)
\end{array}\right.
\end{gathered}
$$

Next, we consider the following conventional test statistic for the unit root test: 


$$
t=\frac{\hat{\rho}-1}{\hat{\sigma}_{\widehat{\rho}}}
$$

where $\hat{\rho}=\frac{\sum_{t=1}^{T} y_{t} y_{t-1}}{\sum_{t=1}^{T} y_{t-1}^{2}}, \hat{\sigma}_{\hat{\rho}}=\left(\hat{\sigma}_{\varepsilon}^{2} / \sum_{t=1}^{T} y_{t-1}^{2}\right)^{1 / 2}, \hat{\sigma}_{\varepsilon}^{2}=T^{-1} \sum_{t=1}^{T}\left(y_{t}-\hat{\rho} y_{t-1}\right)^{2}$.

If the null hypothesis $\rho=1$ holds, then $y_{t}=\sum_{i=1}^{t} u_{t}$ we get

$$
\begin{gathered}
a_{T}^{-2} \sum_{t=1}^{T} y_{t-1} u_{t}=\frac{1}{2}\left[\left(a_{T}^{-1} y_{T}\right)^{2}-a_{T}^{-2} \sum_{t=1}^{T} u_{t}^{2}\right] \stackrel{L}{\rightarrow} \frac{1}{2}\left[U(\tau, 1)^{2}-V(\tau, 1)\right], \\
T^{-1} a_{T}^{-2} \sum_{t=1}^{T} y_{t-1}^{2}=T^{-1} \sum_{t=1}^{T}\left(a_{T}^{-1} y_{t-1}\right)^{2} \Rightarrow \int_{0}^{1} U(\tau, s)^{2} d s
\end{gathered}
$$

Here, since

$$
\begin{aligned}
T(\hat{\rho}-1)=\frac{\sum_{t=1}^{T} y_{t} y_{t-1}}{\sum_{t=1}^{T} y_{t-1}^{2}}-1 & =\frac{\sum_{t=1}^{T} y_{t-1} u_{t}}{\sum_{t=1}^{T} y_{t-1}^{2}} \\
& =\frac{a_{T}^{-2} \sum_{t=1}^{T} y_{t-1} u_{t}}{T^{-1} a_{T}^{-2} \sum_{t=1}^{T} y_{t-1}^{2}} \Rightarrow \frac{\frac{1}{2}\left[U(\tau, 1)^{2}-V(\tau, 1)\right]}{\int_{0}^{1} U(\tau, s)^{2} d s}
\end{aligned}
$$

hold true, the limiting distribution of $t$ test statistic

$$
\begin{aligned}
& \mathrm{t}=\frac{\hat{\rho}-1}{\sigma_{\widehat{\rho}}} \\
& =\frac{a_{T}^{-2} \sum_{t=1}^{T} y_{t-1} u_{t}}{\sqrt{T^{-1} a_{T}^{-2} \sum_{t=1}^{T} y_{t-1}^{2}\left(a_{T}^{-2} \sum_{t=1}^{T}(\hat{\rho}-1)^{2} y_{t-1}^{2}+T^{-1} a_{T}^{-2} \sum_{t=1}^{T} u_{t}^{2}-a_{T}^{-2} \sum_{t=1}^{T} 2\left((\hat{\rho}-1) y_{t-1} u_{t}\right)\right)}} \\
& \stackrel{L}{\rightarrow} \frac{U(\tau, 1)^{2}-V(\tau, 1)}{2 \sqrt{V(\tau, 1) \int_{0}^{1} U(\tau, s)^{2} d s}} \\
& \text { is followed by } a_{T}^{-2} \sum_{t=1}^{T}(\hat{\rho}-1)^{2} y_{t-1}^{2}=o_{p}(1), a_{T}^{-2} \sum_{t=1}^{T} 2\left((\hat{\rho}-1) y_{t-1} u_{t}\right)=o_{p}(1) .
\end{aligned}
$$

\begin{tabular}{|c|c|c|c|c|c|c|c|c|c|c|c|}
\hline \multirow[b]{2}{*}{$\lambda$} & \multirow{2}{*}{$\frac{\kappa}{v}$} & \multicolumn{5}{|c|}{$\mathrm{T}=100$} & \multicolumn{5}{|c|}{$\mathrm{T}=200$} \\
\hline & & 0.1 & 0.3 & 0.5 & 0.7 & 0.9 & 0.1 & 0.3 & 0.5 & 0.7 & 0.9 \\
\hline \multirow[t]{4}{*}{1} & 1.1 & 5.30 & 4.45 & 4.40 & 4.35 & 5.30 & 5.25 & 4.35 & 3.85 & 4.80 & 4.30 \\
\hline & 1.4 & 4.80 & 5.05 & 5.65 & 5.15 & 4.20 & 4.15 & 4.60 & 4.80 & 4.20 & 4.30 \\
\hline & 1.7 & 5.30 & 5.05 & 4.95 & 5.35 & 4.85 & 5.10 & 6.35 & 5.65 & 5.05 & 4.90 \\
\hline & 1.9 & 5.85 & 5.85 & 6.10 & 4.90 & 5.25 & 4.80 & 5.30 & 4.40 & 4.50 & 4.15 \\
\hline \multirow[t]{4}{*}{2} & 1.1 & 5.45 & 6.25 & 6.75 & 6.40 & 5.55 & 4.95 & 4.70 & 5.45 & 6.15 & 5.25 \\
\hline & 1.4 & 5.60 & 6.55 & 6.00 & 7.15 & 4.80 & 5.60 & 5.20 & 5.65 & 5.80 & 5.45 \\
\hline & 1.7 & 5.60 & 6.30 & 6.25 & 7.05 & 5.95 & 6.45 & 6.95 & 7.50 & 6.95 & 6.35 \\
\hline & 1.9 & 6.30 & 7.00 & 7.45 & 5.70 & 6.25 & 5.60 & 6.10 & 7.10 & 6.65 & 5.00 \\
\hline \multirow[t]{4}{*}{4} & 1.1 & 5.20 & 8.00 & 7.70 & 7.80 & 6.15 & 6.05 & 6.25 & 8.35 & 8.30 & 6.60 \\
\hline & 1.4 & 5.75 & 8.05 & 9.10 & 9.05 & 7.20 & 5.60 & 6.55 & 8.75 & 7.95 & 7.80 \\
\hline & 1.7 & 5.55 & 9.65 & 11.60 & 9.80 & 8.30 & 7.65 & 8.70 & 10.45 & 11.55 & 9.00 \\
\hline & 1.9 & 6.55 & 9.65 & 11.00 & 12.90 & 9.50 & 5.50 & 8.45 & 10.65 & 11.50 & 10.15 \\
\hline \multirow[t]{4}{*}{8} & 1.1 & 6.20 & 9.75 & 10.05 & 13.45 & 9.45 & 5.85 & 6.55 & 9.95 & 11.70 & 8.40 \\
\hline & 1.4 & 5.95 & 8.25 & 11.15 & 13.95 & 11.25 & 6.00 & 7.95 & 11.20 & 12.35 & 13.30 \\
\hline & 1.7 & 5.80 & 8.90 & 13.35 & 18.35 & 18.20 & 8.00 & 10.00 & 14.80 & 20.15 & 18.15 \\
\hline & 1.9 & 5.25 & 10.80 & 14.85 & 17.60 & 19.95 & 5.90 & 10.00 & 13.35 & 19.80 & 19.55 \\
\hline
\end{tabular}

\section{Simulation:-}

In this section, we use Monte Carlo simulation method to examine effects of scale volatilities on the size and power. We applied it to the simulated sequence of process (1)-(2) with different values of $\{\kappa=1.1,1.4,1.7,1.9\},\{v=$ $0.1,0.3,0.5,0.7,0.9\}$ and sample sizes $\{T=100,200\}$. For each action, every experiment repeats 2000 times. The significance level is $5 \%$. It takes into account the magnitude of shifts $\lambda=\beta / \alpha$ belonging to $\{1,2,4,8\}$. When $\lambda=1$ there is no scale change. The value of $\rho$ varies among $\{0.5,0.9,0.95,1.0\}$.

Table 1:- Empirical size of $t$ statistic. 
Table 1 reports the empirical size results for $\rho=1.0$. It is expected that the size percentage is close to the significance level if $\lambda=1$. However, as the ratio of scale $\lambda$ increases, the size distortion becomes serious. When $T=$ $100, \kappa=1.4$ and $v=0.5$ and the scale volatility increases from 2 to 8 , and then rejection rate increases, from 6.00 to 11.15. As the sample size increases, substantial over-sizing comes up. Such as, when $\lambda=8, v=0.5$ and $\kappa=1.7$, and the sample size increases from 100 to 200 , and then rejection rate increases, from 13.35 to 14.80 .

It is interesting that the size percentage of $t$ depends on the position of the breakpoint $v$. The size distortion is present except for $v=0.1$. For example, when $T=200, \kappa=1.9$ and $\lambda=4$, the rejection rate is 5.50. With the increase of heavy tail index, the size distortion is severe. Take for example, when $\mathrm{T}=100, v=0.7$ and $\lambda=4$ and the tail index increases from 1.1 to 1.4 and 1.7, and then rejection rate increases, from 7.80 to 9.05 and 9.80 .

Table 2:- Empirical power of $t$ statistic.

\begin{tabular}{|c|c|c|c|c|c|c|c|c|c|c|c|c|c|c|}
\hline & & \multicolumn{10}{c|}{$\mathrm{T}=100$} & \multicolumn{3}{c|}{$\mathrm{T}=200$} \\
\hline & $v$ & \multicolumn{3}{|c|}{$\kappa=1.1$} & \multicolumn{3}{c|}{$\kappa=1.4$} & \multicolumn{3}{c|}{$\kappa=1.4$} \\
\hline$\lambda$ & $\rho$ & 0.5 & 0.9 & 0.95 & 0.5 & 0.9 & 0.95 & 0.5 & 0.9 & 0.95 & 0.5 & 0.9 & 0.95 \\
\hline 1 & 0.1 & 99.90 & 93.75 & 37.95 & 100.00 & 86.55 & 34.55 & 100.00 & 99.70 & 91.90 & 100.00 & 99.65 & 83.95 \\
\hline & 0.3 & 99.90 & 92.85 & 35.95 & 100.00 & 88.00 & 34.50 & 100.00 & 99.55 & 91.60 & 100.00 & 99.65 & 84.60 \\
\hline & 0.5 & 99.90 & 92.90 & 37.70 & 99.90 & 85.95 & 33.75 & 100.00 & 99.55 & 91.60 & 100.00 & 99.60 & 85.55 \\
\hline & 0.7 & 99.95 & 93.25 & 36.95 & 99.95 & 87.85 & 33.60 & 100.00 & 99.40 & 91.85 & 100.00 & 99.70 & 85.05 \\
\hline & 0.9 & 99.90 & 93.40 & 37.45 & 99.85 & 86.00 & 33.40 & 99.95 & 99.60 & 92.15 & 99.90 & 99.75 & 84.35 \\
\hline 2 & 0.1 & 99.80 & 93.65 & 38.55 & 99.90 & 85.00 & 35.80 & 100.00 & 99.55 & 90.55 & 100.00 & 99.65 & 85.00 \\
\hline & 0.3 & 99.70 & 90.65 & 38.00 & 99.85 & 84.95 & 32.80 & 100.00 & 99.50 & 88.45 & 99.90 & 99.30 & 82.65 \\
\hline & 0.5 & 99.70 & 90.90 & 36.65 & 99.95 & 84.65 & 33.90 & 99.80 & 99.50 & 89.15 & 99.95 & 99.10 & 84.20 \\
\hline & 0.7 & 99.70 & 89.80 & 38.60 & 99.85 & 82.05 & 35.25 & 99.90 & 99.20 & 87.90 & 99.95 & 99.00 & 80.85 \\
\hline & 0.9 & 99.50 & 88.85 & 36.75 & 99.50 & 81.00 & 33.50 & 99.95 & 98.85 & 88.45 & 99.95 & 98.50 & 80.65 \\
\hline 4 & 0.1 & 99.80 & 92.55 & 37.30 & 99.90 & 86.85 & 33.25 & 99.95 & 99.85 & 91.25 & 100.00 & 99.45 & 84.75 \\
\hline & 0.3 & 99.80 & 89.85 & 36.90 & 99.95 & 84.15 & 34.65 & 99.90 & 99.35 & 89.40 & 99.95 & 98.90 & 81.40 \\
\hline & 0.5 & 99.85 & 88.30 & 37.10 & 99.80 & 80.10 & 37.30 & 99.80 & 99.30 & 86.30 & 99.85 & 98.85 & 78.90 \\
\hline & 0.7 & 99.65 & 86.15 & 39.40 & 99.45 & 77.10 & 36.75 & 99.95 & 98.40 & 84.15 & 100.00 & 96.90 & 76.55 \\
\hline & 0.9 & 99.20 & 82.95 & 33.55 & 99.25 & 73.00 & 33.10 & 99.70 & 96.75 & 81.05 & 99.90 & 94.40 & 73.25 \\
\hline 8 & 0.1 & 99.85 & 93.20 & 36.05 & 99.95 & 85.90 & 33.00 & 99.95 & 99.40 & 91.55 & 99.95 & 99.65 & 83.30 \\
\hline & 0.3 & 99.90 & 88.90 & 36.20 & 99.85 & 82.10 & 34.15 & 99.85 & 99.40 & 87.65 & 100.00 & 99.30 & 80.40 \\
\hline & 0.5 & 99.55 & 85.95 & 39.10 & 99.75 & 78.40 & 38.50 & 99.90 & 99.00 & 85.00 & 100.00 & 98.70 & 79.05 \\
\hline & 0.7 & 99.35 & 83.50 & 40.90 & 99.65 & 75.50 & 42.30 & 99.90 & 97.40 & 81.25 & 99.90 & 96.85 & 72.25 \\
\hline & 0.9 & 99.05 & 75.70 & 35.60 & 98.25 & 66.00 & 38.95 & 99.40 & 93.30 & 73.10 & 99.60 & 90.00 & 65.85 \\
\hline
\end{tabular}

Table 2:- Empirical power of $t$ statistic.

\begin{tabular}{|l|l|l|l|l|l|l|l|l|l|l|l|l|l|l|}
\hline & \multicolumn{10}{|l|}{$\mathrm{T}=100$} & \multicolumn{3}{|c|}{$\mathrm{T}=200$} \\
\hline & $v$ & \multicolumn{3}{|c|}{$\kappa=1.7$} & \multicolumn{3}{c|}{$\kappa=1.9$} & \multicolumn{3}{c|}{$\kappa=1.9$} \\
\hline$\lambda$ & $\rho$ & 0.5 & 0.9 & 0.95 & 0.5 & 0.9 & 0.95 & 0.5 & 0.9 & 0.95 & 0.5 & 0.9 & 0.95 \\
\hline 1 & 0.1 & 100.00 & 82.90 & 36.40 & 99.95 & 82.30 & 33.00 & 100.00 & 99.85 & 83.20 & 100.00 & 99.90 & 76.75 \\
\hline & 0.3 & 99.95 & 81.70 & 33.85 & 100.00 & 79.70 & 34.80 & 100.00 & 99.80 & 84.10 & 100.00 & 99.65 & 76.65 \\
\hline & 0.5 & 100.00 & 82.50 & 33.90 & 100.00 & 80.00 & 35.30 & 100.00 & 99.95 & 84.20 & 100.00 & 99.70 & 76.20 \\
\hline & 0.7 & 100.00 & 83.20 & 34.85 & 100.00 & 80.00 & 33.90 & 100.00 & 99.90 & 83.15 & 100.00 & 99.70 & 77.35 \\
\hline & 0.9 & 100.00 & 82.45 & 34.45 & 100.00 & 81.55 & 34.20 & 100.00 & 99.60 & 85.40 & 100.00 & 99.80 & 78.75 \\
\hline 2 & 0.1 & 100.00 & 81.55 & 35.15 & 100.00 & 82.10 & 35.05 & 99.95 & 99.75 & 83.65 & 100.00 & 99.80 & 77.15 \\
\hline & 0.3 & 99.85 & 79.45 & 36.20 & 99.95 & 76.45 & 34.40 & 100.00 & 99.35 & 81.80 & 100.00 & 99.55 & 75.35 \\
\hline & 0.5 & 99.90 & 78.25 & 36.60 & 99.95 & 77.75 & 35.60 & 100.00 & 99.50 & 80.35 & 100.00 & 98.70 & 73.40 \\
\hline & 0.7 & 100.00 & 77.65 & 36.20 & 99.95 & 74.85 & 37.45 & 100.00 & 98.75 & 77.00 & 100.00 & 98.35 & 72.60 \\
\hline & 0.9 & 99.85 & 74.50 & 33.30 & 100.00 & 73.40 & 35.15 & 100.00 & 98.35 & 76.90 & 100.00 & 96.80 & 70.85 \\
\hline \hline 4 & 0.1 & 100.00 & 82.35 & 36.45 & 100.00 & 79.90 & 36.55 & 100.00 & 99.60 & 84.35 & 100.00 & 99.60 & 77.10 \\
\hline & 0.3 & 99.95 & 78.40 & 36.55 & 100.00 & 76.95 & 39.25 & 100.00 & 99.40 & 80.75 & 100.00 & 99.45 & 75.05 \\
\hline & 0.5 & 99.95 & 74.50 & 38.95 & 100.00 & 73.45 & 38.40 & 100.00 & 99.00 & 77.75 & 100.00 & 98.20 & 70.65 \\
\hline & 0.7 & 99.85 & 72.80 & 43.50 & 100.00 & 71.90 & 40.85 & 100.00 & 96.70 & 74.80 & 100.00 & 95.00 & 71.30 \\
\hline
\end{tabular}




\begin{tabular}{|l|l|l|l|l|l|l|l|l|l|l|l|l|l|}
\hline & 0.9 & 99.50 & 69.05 & 39.75 & 99.60 & 67.90 & 40.15 & 99.95 & 91.90 & 71.85 & 99.95 & 88.95 & 64.85 \\
\hline 8 & 0.1 & 99.95 & 80.40 & 35.20 & 100.00 & 79.30 & 34.30 & 100.00 & 99.65 & 83.05 & 100.00 & 99.60 & 76.20 \\
\hline & 0.3 & 99.95 & 77.95 & 38.10 & 100.00 & 77.25 & 38.15 & 100.00 & 99.50 & 79.85 & 100.00 & 99.05 & 73.90 \\
\hline & 0.5 & 99.95 & 74.05 & 39.65 & 100.00 & 74.65 & 40.05 & 100.00 & 98.15 & 75.65 & 100.00 & 98.20 & 72.25 \\
\hline & 0.7 & 99.70 & 72.60 & 45.75 & 99.95 & 70.90 & 45.15 & 100.00 & 96.15 & 71.55 & 100.00 & 92.85 & 68.90 \\
\hline & 0.9 & 98.20 & 63.35 & 46.30 & 97.60 & 64.70 & 48.25 & 99.75 & 84.80 & 64.05 & 99.80 & 81.90 & 63.60 \\
\hline
\end{tabular}

Now, the consequences for the empirical power are collected in Table 2-3. It is not surprised that the reject power enhances as $\rho$ is far away from 1. Second, the larger ratio of scales does induce the smaller reject percentage. For instance, when $T=100, v=0.5, \rho=0.95$ and $\kappa=1.7$, the rejection rate is 82.50 to 74.50 and 74.05 for $\lambda=$ $1,4,8$. Third, the reject rate decrease as the location of breakpoint closes to the endpoint. Such as, when $T=$ $200, \kappa=1.7, \lambda=8$ and $\rho=0.95$, the breakpoint position increases from 0.1 to 0.5 and 0.9 , then power decreases from 83.05 to 75.65 and 64.05 . Forth, the reject power increase with samples size approach to infinite. Finally, the rejection frequency is still sensitive to the heavy tail index. For example, when $T=100, v=0.3, \rho=0.9$ and $\lambda=$ 2 , and the tail index increases from 1.1 to 1.4 and 1.9, then rejection rate drops, from 90.05 to 84.95 and 76.45 .

\section{Conclusion:-}

This paper examines the unit root test for heavy tailed series with single change point in scale volatility. The asymptotic distribution of conventional test statistic was proved and the simulations on finite samples report that non-constant scales volatility can inflate the rejection frequency of the test. Thus, standard unit root test is invariant to structural changes in scales volatility. The size distortion becomes large as the time of scale shift moves backwards.

\section{Acknowledgements:-}

Financial support from NNSF (No.71103143, 71273206, 71473194) is gratefully acknowledged. In addition, the authors state that there is no conflict of interest regarding the publication of this article.

\section{References:-}

1. Agnieszka Jach, Piotr Kokoszka, Subsampling unit root tests for heavy-tailed observations[J], Methodologyand computing in Applied Probability, 2003, 6: 73-97;

2. Dickey, D.A and W.A Fuller., Distribution of estimators for autoregressive time series with a unit root [J]. Journal of the American Statistical Association, 1979, 74:427-431;

3. Dickey, D.A and W.A Fuller., Likelihood ratio statistics for autoregressive-moving average models of unknown order [J]. Biometrika, 1984, 71: 599-607;

4. Fuller, W. A., Hasza, D. P., and Goebel, J. J., Estimation of the parameters of stochastic difference equations [J], The Annals of Statistics, 1981 ,9:531-543;

5. Giuseppe Cavaliere, Unit root under time-varying variances [J], Econometric Reviews, 2004,23(3):259-292;

6. Guodong Li, Chenlei Leng, Chih-ling Tsai. A hybrid bootstrap approach to unit root tests. Journal of Time Series Analysis. 2014,35:299-321;

7. Hamori, S., Tokihisa, A., Testing for a unit root in the presence of a variance shift [J]. Econ. Lett. 1997, 57:245253;

8. Jesus Clemente, Antonio Mointanes,Marcelo Reyes, Testing for a unit root in variance with a double change in the mean [J], Economics Letters, 1998,59:175-182

9. Joakim Westerlund. On the choice of test for a unit root when the errors are conditionally heteroskedastic. Computational Statistics and Data Analysis. 2014,89:40-53;

10. Kokoszka, P., Wolf, M., Subsampling the mean of heavy-tailed dependent observations [J], Time Series Anal, 2004, 25:217-234;

11. Ngai Hang Chan, Lanh Tat Tran. On the first order autoregressive process with infinite variance [J]. Econometric Theory. 1989,5:354-362;

12. Rolf Larsson, Johan Lyhagen. Likelihood Ratio Tests for a Unit Root in Panels with Random Effects [J]. Deakin University School Working Paper.2015;

13. Lumsdaine, R.L., Papell, D.H., 1997. Multiple trends and the unit root hypothesis [J]. The Review of Economics and Statistics, 1997. 79: 212-218.

14. Perron, P., Vogelsang, T. Nonstationarity and level shifts with an application to purchasing power parity [J]. Journal of Business and Economic Statistics, 1992, 10: 301-320; 
15. Phillips P.C.B., Time series regression with a unit root. Econometric, 1987, 55:277-301;

16. Phillips P.C.B., Perron P, Testing for a unit root in time series regression [J]. Biometrika, 1989, 75:355-346;

17. Resnick, S. I. Point process, regular variation and weak convergence [J]. Adv. Appl. Probab.1989,18:66-138;

18. Ryota Yabe. Asymptotic distribution of the conditional-sum-of-squares estimator under moderate deviation from a unit root in MA(1) [J]. Statistics and Probability Letters, 2017,125:220-226;

19. Said, S. E., and Dickey, D. A., Testing for unit roots in autoregressive-moving average models of unknown order, Biometrika, 1984,71:599-608;

20. Stephen J. Leybourne, Paul Newbold, Behavior of the standard and symmetric Dickey-Fuller-type tests when there is a break under the null hypothesis [J], Econometrics Journal, 2000, 3:1-15;

21. Stelios Arvanitis. A note on the limit theory of a Dickey-Fuller unit root test with heavy tailed innovations. Statistics and Probability Letters. 2017;

22. Stephen J. Leybourne, Paul Newbold, Behavior of Dickey-Fuller t-tests when there is a break under the alternative hypothesis [J], Econometrics Theory, 2000, 16:779-789; 